\title{
Justifying referrals for paediatric CT
}

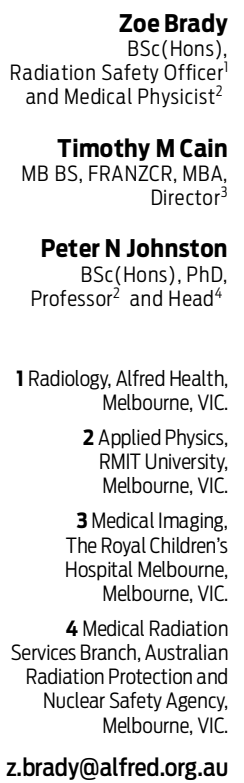

MJA 2012; 197: 95-98 doi: 10.5694/mjall.11124 $\mathrm{n}$ adolescent female patient presents with acute lower back pain of recent onset and no history of trauma. Is it warranted to order a computed tomography (CT) scan of her lumbar spine? The justification for a CT scan at the individual level is complex and the risk-benefit ratio must be considered; the risks associated with radiation are often not well understood and, similarly, the benefit for an individual may be difficult to determine.

Adverse outcomes of CT scans (in addition to radiationinduced cancer) may include false-positive scan results, unnecessary treatment due to overdiagnosis, and anxiety for the patient or their family. Benefits can be significant and include diagnosis, treatment, changes to management, and disease surveillance. Even a null finding on a CT scan may provide emotional relief and exclude conditions requiring treatment. Furthermore, as the Royal Australian and New Zealand College of Radiologists (RANZCR) states: "the risks involved in failing to have a needed medical imaging test include potential missed diagnosis and delayed, inappropriate or lack of necessary treatment" ${ }^{1}$

At a population level, the risk-benefit ratio may be more readily assessed. For example, there is justification for mammographic screening of women in target age groups, based on the net reduction in breast cancer mortality. 2,3 Conversely, the benefits of full-body CT scanning in healthy adults are not established. ${ }^{4}$ In Australia, RANZCR does not support the use of diagnostic imaging for screening of asymptomatic patients, except "where there is an evidence-base to support the screening program". ${ }^{5}$ National professional bodies provide justification at this higher level, but when considering the individual patient, it is the doctor who makes the risk-benefit analysis.

In years to come, the overuse of higher dose imaging modalities such as CT may lead to an unreasonable population health burden from radiation-induced cancers. It is essential that medically indicated CT scans are performed when the potential detrimental impact on management and diagnosis of not performing the scan outweighs any small theoretical radiation risk. ${ }^{1,6,7}$ However, it is also important not to discount the potential radiation risks, particularly cumulative effects. ${ }^{7,8}$ These are more significant for the younger population, for whom the risks are higher and there is more opportunity for repeated imaging.

Our review aims to provide referrers with additional information about radiation and risk when considering the risk-benefit ratio for diagnostic imaging. Although focusing on paediatric $\mathrm{CT}$, the general principles and content apply more broadly to any request for imaging involving radiation exposure.

\section{Radiation and risk}

The exposure risk from diagnostic medical imaging is small. However, it is assumed that this risk is cumulative

\section{Summary \\ - The system of radiation protection assumes a linear dose-response relationship with no threshold for low doses and dose rate exposures. This is based on epidemiological evidence at higher doses. \\ - Hence there is a small theoretical risk of carcinogenesis attributable to low doses of ionising radiation. This risk is associated with any diagnostic imaging procedure involving radiation. \\ - Radiosensitivity declines with age, so children are more susceptible to radiation risks than adults. Females are more radiosensitive than males. \\ - The radiation protection system is based on the assumption that radiation risk is cumulative over a lifetime. \\ - For an individual, a justified, optimised computed tomography (CT) scan will result in more benefit than harm. A doctor must justify the necessity for a CT scan before referring an individual for imaging.}

over a lifetime, as the mechanisms for adaptive cellular responses to low doses of radiation remain unclear. ${ }^{9-11}$ There are considerable uncertainties in estimating radiation risk at low doses, and this leads to a range of interpretations about risk, as shown by recent commentary in this Journal on the use of CT. ${ }^{6-8,12}$

There is irrefutable evidence from epidemiological studies that ionising radiation exposure at high doses is associated with an increase in cancer incidence and morbidity. ${ }^{13}$ However, quantifying the risk of a low dose (the dose from a single CT scan is below $100 \mathrm{mSv}$ ) is complex and contentious. Studies of populations exposed to these low doses are inherently difficult to collate, due to the need for sufficient sample sizes and extensive follow-up to account for radiation-induced cancer latency. ${ }^{9,10,14}$ It is extremely difficult to observe the weak carcinogenic effects of radiation above the high natural incidence of cancer with any statistical significance and, consequently, the risks at very low levels of exposure may never be precisely known. ${ }^{10,14}$ Despite these limitations, observational epidemiological studies largely remain the basis for radiation risk modelling.

The most important epidemiological study is the JapanUnited States cooperative Life Span Study (LSS) of Japanese atomic bomb survivors, because of the large exposed cohort, including men, women and children, and the extensive long-term follow-up that has been undertaken. ${ }^{13}$ The most widely accepted model of low-dose risk estimation using current scientific evidence is based predominantly (but not solely) on LSS data, with a linear extrapolation of the risks at high doses with no threshold. ${ }^{9,11,15}$ Arguments against using this model continue, ${ }^{16}$ and there is some evidence of protective effects from low doses of radiation, or a hormetic response, but these are yet to be confirmed through epidemiological studies. ${ }^{17}$ 


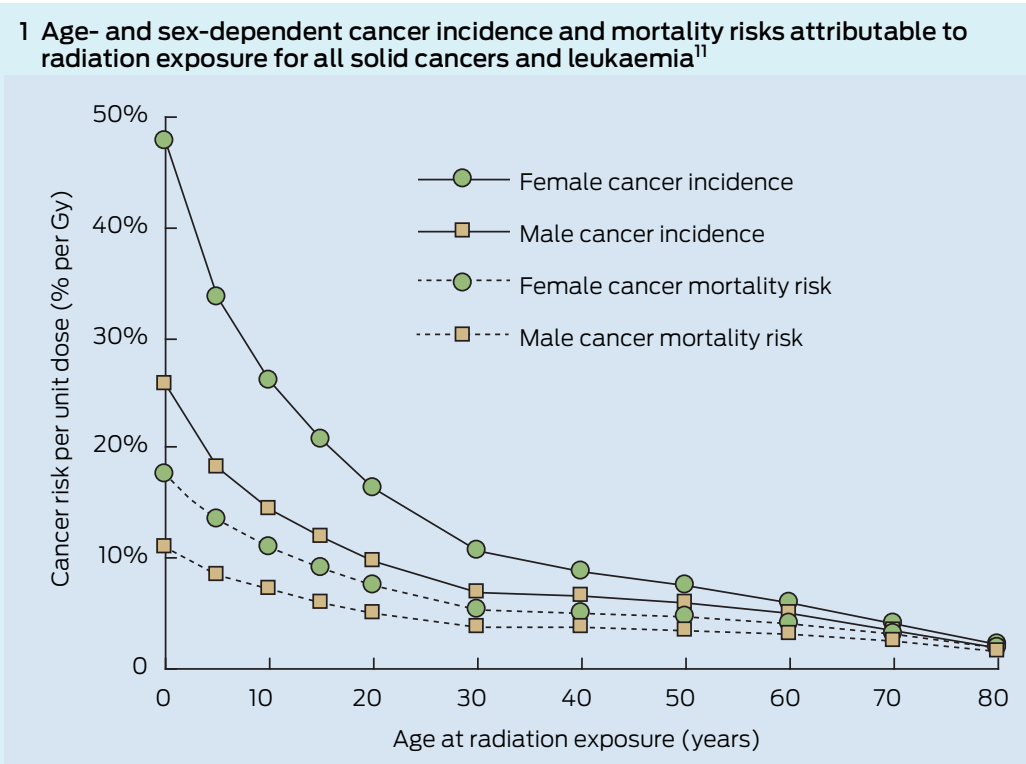

Furthermore, there is no observable increase in cancer incidence in areas of high background radiation such as Kerala, India. ${ }^{18}$

The first epidemiological link between cancer risk and low doses of radiation from CT scans performed on children and young adults has been published very recently. These data strongly support a linear dose-response model and no threshold in the low dose region. The risks are broadly consistent with those recommended in the international system of radiation protection. ${ }^{9,11}$ Despite the lack of extensive epidemiological evidence, the linear nothreshold (LNT) model remains the most reasonable assumption regarding the dose-response relationship at low levels. ${ }^{9}$

A framework for estimating the theoretical lifetime attributable risk (LAR) due to low radiation exposures is provided in the Biological Effects of Ionising Radiation (BEIR) VII report. ${ }^{11}$ The risk estimation predominantly relies on LSS data, although other medically exposed cohorts were also taken into account for assessment of breast and thyroid cancer development. Considerable uncertainties exist in the BEIR VII methodology, due to extrapolation of LSS data to low total dose and low dose rate exposure situations, transporting the risks from the Japanese population to other ethnicities, and sampling variability in the model parameter estimates for the LSS data. However, the age, sex and site-dependent LARs are the best method currently available for estimating the detriment from exposure situations in which doses differ substantially across the body, as is the case for medical imaging exposures.

In the BEIR VII report, rates for all radiation-attributable cancer incidence are about twice the rates for mortality. ${ }^{11}$ Risk is dependent on sex and the age at which exposure occurred (Box 1). Children are considerably more susceptible to radiation than adults because their cells are more rapidly dividing and not yet mature, and they have more years of remaining lifetime in which a radiation-induced cancer may appear. For example, the risk of radiationinduced fatal cancer from the same magnitude of exposure for a 10-year-old girl is double the risk for a 30-year-old woman, and more than five times the risk for an 80 -yearold woman. However, a recent study ${ }^{20}$ suggests that the risks in middle age may have been underestimated, and this may warrant consideration in the future.

Defining what is acceptable in terms of risk will always be challenging. Human nature tends towards overreaction when presented with a small risk, while larger risks, such as dying from lung cancer due to smoking, are often downplayed. ${ }^{21}$ It is complex to justify imaging, because it potentially provides immediate benefit for a current health problem, while the radiation effects are possible future risks.

\section{Paediatric CT}

In countries with advanced health care systems, the number of diagnostic examinations performed each year that use ionising radiation is greater than one per person. ${ }^{22}$ In Australia, the number of CT examinations is increasing by about $9 \%$ every year (Box 2), and while not showing the same rate of growth, the CT imaging rate for children is also increasing. ${ }^{23}$ The first decrease in CT imaging in more than 15 years occurred in 2010. This is promising, but cannot yet be confirmed as an ongoing trend. Magnetic resonance imaging (MRI) demonstrates continued growth over recent years (Box 2). The increase in CT use can be partly attributed to advances in technology which allow faster scanning times, and hence greater feasibility of using CT for scans on uncooperative, young and/or sick patients.

In 2001, a series of articles ${ }^{24-26}$ generated considerable media coverage in the US about the risks to children from CT scanning. An important realisation then was that CT protocols for adults were sometimes being used on children, with no appreciable adjustment of parameters based on the examination type or patient age. ${ }^{24}$

As a result of an increased focus on paediatric $\mathrm{CT}$, an Alliance for Radiation Safety in Pediatric Imaging was

\section{Number of computed tomography (CT) and magnetic resonance imaging (MRI) services billed to Medicare Australia from 1994 to 2010}

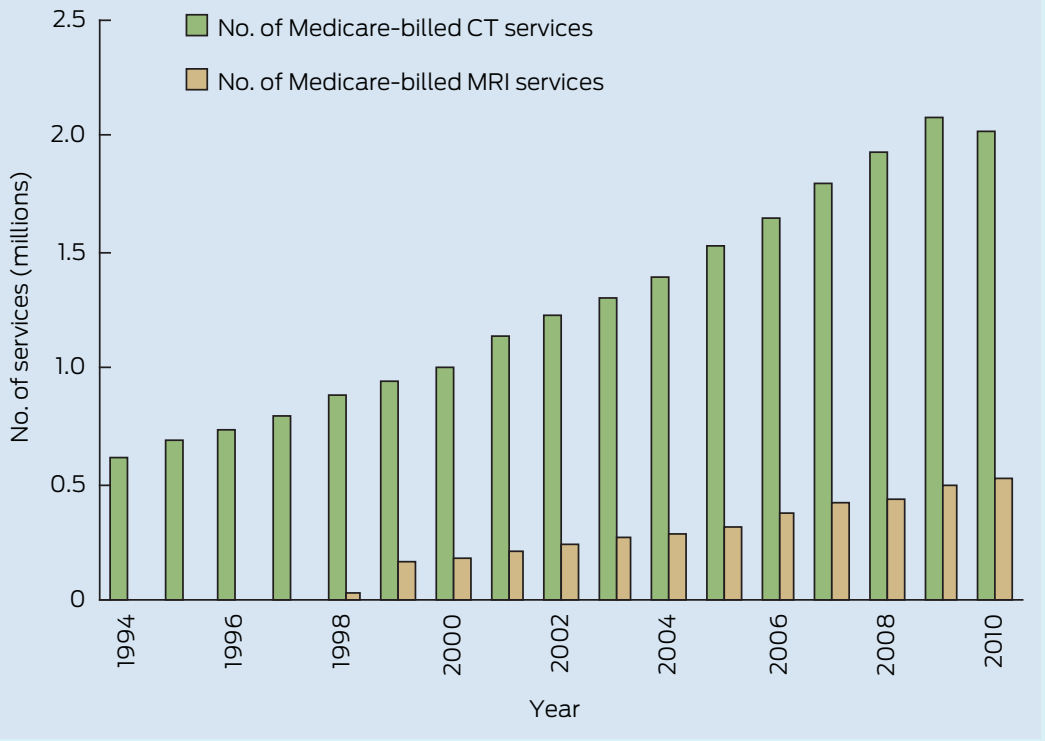




\begin{tabular}{|c|c|c|c|}
\hline \multirow[b]{2}{*}{ Age group } & \multicolumn{3}{|c|}{ Examination } \\
\hline & Brain CT & Chest CT & Abdominal/pelvic CT \\
\hline$<5$ years & $1.5(75) *$ & $2.1(105) *$ & $3.3(165) *$ \\
\hline 5-10 years & $1.4(70) *$ & $5.4(270) *$ & $6.3(315) *$ \\
\hline$>10$ years & $1.0(50) *$ & 6.7 (335)* & $4.7(235) *$ \\
\hline
\end{tabular}

$\mathrm{CT}=$ computed tomography. $*$ Equivalent number of posterior-anterior chest $\mathrm{x}$-rays for an adult at $0.02 \mathrm{mSv}$ doses.

formed in $2007,{ }^{27}$ with RANZCR subsequently becoming a member. ${ }^{1}$ The key recommendations of the Alliance, through their Image Gently campaign, are to scan only when necessary; reduce or "child-size" the amount of radiation used; scan only the indicated region; and scan once (multiphase scanning is usually not necessary in children). ${ }^{27,28}$ These points capture the basis for dose reduction in paediatric $\mathrm{CT}$ and the radiation protection principles of optimisation and justification. The referrer can influence the process of optimisation, although it is predominantly the responsibility of the practice undertaking the examination and involves a multidisciplinary team of radiologists, radiographers and physicists. The initial justification for the scan is the responsibility of the referrer.

\section{Justifying the scan}

A CT examination for an individual child is justified if there is a reasonable likelihood that it will produce a health benefit or inform patient management. Otherwise, the child only receives the potential detrimental radiation effects. The population obtains an advantage when the summed positive health benefits from those whose management is positively influenced by the imaging are greater than the summed detrimental effects. The population also benefits from the ability of diagnostic imaging to identify who may need health intervention, and therefore allow appropriate allocation of health resources. The net positive effects of the CT examination will be greater when the referring doctor applies the principle of justification to ensure unnecessary studies are not performed. ${ }^{9,29}$ Further net population benefit will be achieved when the studies performed are limited to the required body region and optimised for image quality and dose.

The principle of justification applied to medical radiation exposure situations requires the referring doctor (and radiologist) to weigh the potential benefits of the procedure against the risks of radiation detriment to ensure that there is a net population benefit. ${ }^{9,28}$ The doctor should consider the objective of the exposure and the individual involved. Prior imaging for the individual should be checked to see if the required diagnostic information has already been acquired. Alternative procedures that do not involve ionising radiation, such as MRI or ultrasound, should also be considered, especially for follow-up imaging.

There are numerous reasons why unnecessary scans may be undertaken, including defensive medicine (fear of litigation from not performing a CT scan); pressure from the public to use "high-tech" examinations; greater availability and access to CT compared with non-ionising radiation modalities; and, in some cases, there is financial incentive to perform more CT scans. ${ }^{30,31}$ The Professional Services Review in Australia recently reported that CT scans were being initiated without appropriate clinical justification. ${ }^{32}$ The report suggested that lack of knowledge about particular imaging modalities and using imaging instead of adequate clinical assessment (due to time pressure) may also contribute to inappropriate CT usage. ${ }^{32}$

The International Commission on Radiological Protection (ICRP) states that justification is the shared responsibility of the requesting clinician and the radiologist. ${ }^{29}$ If opportunities are not used to educate referring doctors about inappropriate requests, then this lack of knowledge may also contribute to the growth in CT use. ${ }^{31}$ The referral process may be supported by clinical decision rules and online decision-making tools such as Diagnostic Imaging Pathways, developed and maintained by the Western Australian Department of Health (http://www.imagingpathways.health.wa.gov.au). RANZCR have also established InsideRadiology, a consumer and referrer information database on medical imaging to encourage informed choice (http://www.insideradiology.com.au). ${ }^{8}$

It has been suggested that informed consent be obtained for children and young adults undergoing CT examinations. ${ }^{12}$ Other authors comment on the difficulty of obtaining consent, because the radiation risk is age dependent and cumulative, ${ }^{33}$ or because of the possibility of a parent refusing a justified CT scan. ${ }^{34}$ One study surveyed parents of children undergoing CT scans, before and after providing a brochure on radiation safety, and found that the rates of consent to CT scans did not change. ${ }^{35}$ Informed decision making and support resources may be a viable alternative to informed consent. ${ }^{33}$ Box 3 provides typical effective doses for paediatric CT examinations with the number of equivalent chest radiographs given as a radiation dose comparator. While it may be desirable to quote a " 1 -in- $x^{\text {" }}$ risk for each type of examination, these types of estimates are not applicable to effective doses. Risk can only be determined from organ-absorbed doses using risk coefficients specific to anatomy, age and sex.

Box 4 summarises points that a doctor should consider when requesting a CT scan.

\section{Conclusions}

The system of radiation protection is based on the extrapolation of radiation exposure risks at higher doses to the lower doses that are typical of CT imaging, which means that even low doses of ionising radiation are potentially harmful, particularly for children. It will require large-scale, long-term epidemiological studies of CT scan recipients to quantify the risk. The first of many epidemiological studies $^{19}$ has appeared and supports the assumptions regarding risk, which are used as the basis of radiation protection.

In the past decade, recognition of the potential radiation risks combined with the high use of CT imaging has led to greater awareness of population health risks. These risks can be reduced through education to avoid unnecessary 


\section{Points for consideration when a doctor requests a} computed tomography scan

There are several factors that referring doctors should consider when requesting a computed tomography (CT) scan, particularly for children and young adults:

- Is the scan really needed?

- Will it change management and/or diagnosis, taking into account the severity of the condition?

- Is there previous appropriate imaging available?

- Can the same information be obtained without using ionising radiation (eg, magnetic resonance imaging or ultrasound), particularly for follow-up imaging?

- Has information from a clinical decision-support tool, (eg, Diagnostic Imaging Pathways) been considered?

- Is enough clinical information provided to ensure the correct examination is undertaken?

Questions to ask the practice carrying out the imaging:

- Do you have an active optimisation program for radiation dose and image quality?

- Do you have local diagnostic reference levels for CT examinations?*

Are the radiation doses from medical imaging kept as low as reasonably achievable (the ALARA principle) and are protocols modified for follow-up imaging?

Are technique factors tailored to paediatric patients?

* Australian national diagnostic reference levels are available for adult

CT scans at http://www.arpansa.gov.au/services/ndrl/adult.cfm

imaging and by ensuring that CT scans are performed with an optimal technique.

Returning to the question about justification of a lumbar spine CT scan for a young female patient with acute lower back pain: if there are no red flags, then conservative management should be trialled first. ${ }^{31,32}$ Exposure risks associated with diagnostic medical imaging using ionising radiation are unavoidable, but these risks can be appropriately managed and reduced.

Competing interests: No relevant disclosures.

Provenance: Not commissioned; externally peer reviewed.

1 Royal Australian and New Zealand College of Radiologists. Computed Tomography and Radiation Risks. Position statement. Sydney: RANZCR, 2010. http://www.ranzcr.edu.au/resources/professional-documents/positionstatements (accessed Jun 2012)

2 de Gelder R, Draisma G, Heijnsdijk EA, de Koning HJ. Population-based mammography screening below age 50: balancing radiation-induced vs prevented breast cancer deaths. Br J Cancer 2011; 104: 1214-1220.

3 Yaffe MJ, Mainprize JG. Risk of radiation-induced breast cancer from mammographic screening. Radiology 2011; 258: 98-105.

4 Brenner DJ, Elliston CD. Estimated radiation risks potentially associated with full-body CT screening. Radiology 2004; 232: 735-738.

5 Royal Australian and New Zealand College of Radiologists. The use of diagnostic imaging for screening purposes and non-referred investigations. Statement of principles. Sydney: RANZCR, 2005. http://www.ranzcr.edu.au/ resources/professional-documents/policies (accessed Jun 2012).

6 Blecher CM. Alarm about computed tomography scans is unjustified [letter]. Med J Aust 2010; 192: 723-734.

7 Mendelson RM, Fox RA, de Klerk NH. Alarm about computed tomography scans is unjustified [letter]. Med J Aust 2010; 193: 246.

8 Heggie JC, Goergen SK, Fallon MJ. Alarm about computed tomography scans is unjustified [letter]. Med J Aust 2011; 194: 150-151.
9 International Commission on Radiological Protection. The 2007 recommendations of the International Commission on Radiological Protection. ICRP Publication 103. Ann ICRP 2007; 37: 1-332.

10 International Commission on Radiological Protection. Low-dose extrapolation of radiation-related cancer risk. ICRP Publication 99. Ann ICRP 2005: 35: 1-140.

11 National Research Council. Health risks from exposure to low levels of ionizing radiation: BEIR VII Phase 2. Washington, DC: The National Academies Press, 2006. http://www.nap.edu/catalog.php?record_id=11340 (accessed Jun 2012).

12 de Campo JF, de Campo MP. Is informed consent necessary for computed tomography in children and young adults? [letter]. Med J Aust 2010; 192: 423.

13 Preston DL, Shimizu Y, Pierce DA, et al. Studies of mortality of atomic bomb survivors. Report 13: Solid cancer and noncancer disease mortality: 1950-1997. Radiat Res 2003; 160: 381-407.

14 Brenner DJ, Doll R, Goodhead DT, et al. Cancer risks attributable to low doses of ionizing radiation: assessing what we really know. Proc Natl Acad Sci U SA 2003; 100: 13761-13766.

15 United Nations Scientific Committee on the Effects of Atomic Radiation. Sources and effects of ionizing radiation. Vol l: Sources. Vol II: Effects. UNSCEAR 2000 Report to the General Assembly, with scientific annexes. New York: United Nations, 2000. http://www.unscear.org/unscear/en/publications.html (accessed Jun 2012).

16 Tubiana M, Feinendegen LE, Yang C, Kaminski JM. The linear no-threshold relationship is inconsistent with radiation biologic and experimental data. Radiology 2009; 251: 13-22.

17 Sykes PJ, Day TK. Requirements for identification of low dose and non-linear mutagenic responses to ionising radiation. Dose Response 2007; 5: 308-314.

18 Nair RR, Rajan B, Akiba S, et al. Background radiation and cancer incidence in Kerala, India - Karanagappally cohort study. Health Phys 2009; 96: 55-66.

19 Pearce MS, Salotti JA, Little MP, et al. Radiation exposure from CT scans in childhood and subsequent risk of leukaemia and brain tumours: a retrospective cohort study. Lancet 2012; 7 Jun. [Epub ahead of print.] doi: 10.1016/S01406736(12)60815-0.

20 Shuryak I, Sachs RK, Brenner DJ. Cancer risks after radiation exposure in middle age. J Natl Cancer Inst 2010; 102: 1628-1636.

21 Picano E. Informed consent and communication of risk from radiological and nuclear medicine examinations: how to escape from a communication inferno. BMJ 2004; 329: 849-851.

22 United Nations Scientific Committee on the Effects of Atomic Radiation. Sources and effects of ionizing radiation. Vol I. UNSCEAR 2008 Report to the UN General Assembly. Annex A: Medical Radiation Exposures. New York: United Nations, 2010. http://www.unscear.org/unscear/en/publications.html (accessed Jun 2012)

23 Brady Z, Cain TM, Johnston PN. Paediatric CT imaging trends in Australia. J Med Imaging Radiat Oncol 2011; 55: 132-142.

24 Paterson A, Frush DP, Donnelly LF. Helical CT of the body: are settings adjusted for pediatric patients? AJR Am J Roentgenol 2001; 176: 297-301.

25 Brenner D, Elliston C, Hall E, Berdon W. Estimated risks of radiation-induced fatal cancer from pediatric CT. AJR Am J Roentgenol 2001; 176: 289-296.

26 Donnelly LF, Emery KH, Brody AS, et al. Minimizing radiation dose for pediatric body applications of single-detector helical CT: strategies at a large Children's Hospital. AJR Am J Roentgenol 2001; 176: 303-306.

27 Goske MJ, Applegate KE, Boylan J, et al. The Image Gently campaign: working together to change practice. AJR Am J Roentgenol 2008; 190: 273-274.

28 Image Gently. The Alliance for Radiation Safety in Pediatric Imaging. http:// www.pedrad.org/associations/5364/ig/ (accessed Jun 2012).

29 International Commission on Radiological Protection. Radiation protection in medicine. ICRP Publication 105. Ann ICRP 2007; 37: 1-63.

30 Donnelly LF. Reducing radiation dose associated with pediatric CT by decreasing unnecessary examinations. AJR Am J Roentgenol 2005; 184: 655-657.

31 Hendee WR, Becker GJ, Borgstede JP, et al. Addressing overutilization in medical imaging. Radiology 2010; 257: 240-245.

32 Professional Services Review. PSR report to the professions 2008-09. Canberra: Commonwealth of Australia, 2010. http://www.psr.gov.au/publications-andresources/other-publications (accessed Jun 2012).

33 Goske MJ, Bulas D. Improving health literacy: informed decision-making rather than informed consent for CT scans in children. Pediatr Radiol 2009; 39: 901-903.

34 Karsli T, Kalra MK, Self JL, et al. What physicians think about the need for informed consent for communicating the risk of cancer from low-dose radiation. Pediatr Radiol 2009: 39: 917-925.

35 Larson DB, Rader SB, Forman HP, Fenton LZ. Informing parents about CT radiation exposure in children: it's OK to tell them. AJR Am J Roentgenol 2007; 189: 271-275. 
Clinical focus

Aus Med Handbook AMA 